\title{
Beitrag \\ zur Kenntnis des Benzidins als Chromogen bei den biologischen Oxydationsreaktionen.
}

Von

Dr. med, et med. vet. M. Kjöllerfeldt aus Helsingfors.

(Aus dem Instint für physikalisch-chemische Biologie der Universität Bern.)

Anlässlich einer A $A$ beit über die Oxydationsfermente des Blutes, die ich auf Anregung der Leiterin des Instituts für physikalischchemische Biologie in Bern, Fräulein Dr. W oker, im Sommersemester 1915 ausführte, machte ich über das Benzidin, das ich als Chromogen bei den Oxydationsreaktionen verwendete, eine Reihe Beobachtungen, die eine grosse Beeinflussbarkeit der Farbenreaktion dartaten. Dies veranlasste mich, mich näher mit der Konstitution des Benzidins zu befassen in der gleichzeitigen Absicht, ein für die biologischen Oxydationen quantitativ brauchbares und konstantes Reagens zu erlangen.

Meine Bemühungen in der angegebenen Richtung. nebst den dabei gemachten Beobachtungen sind in der oben erwähnten Arbeit zusammengestellt. Das Benzidin ist als Reagens auf Oxydationsfermente zuerst von 0 . und R. Adler ${ }^{1}$ ) eingeführt worden. In einer Arbeit über die Methoden zum Nachweis des Blutes hahen die Autoren das Verhalten aromatischer Amine, Phenole, aromatischer Säuren und Verbindungen der Diphenyl- und der Naphthalingruppe zum Blit + Peroxyd systematiseh untersucht. Sie geniessen auch das Prioritätsrecht, das Benzidin in alkoholischer Lösung als Farbenreagens auf Blut eingeführt zu haben.

Sie erklärten die Farbenreaktion des Benzidins mit Blut + Peroxyd als eine Oxydation, wobei das Blut der Vermittler zwischen dem oxydationsfäligen Körper, dem Benzidin und dem indirekten Oxydations-

1) O. und R. Adler, Über das Verhalten gewisser organischer Verbindungen gegenüber Blut mit besonderer Berücksichtigung des Nachweises von Blut. Zeitschr. f. physiol. Chemie Bd. 41 S. 59. 1904. 
mittel, dem Peroxyd, sei. Als Vermittler diese: Oxydationsvorgangs konnten an Stelle des Blutes auch Eisenoxydulsalze, Rhodansalze und ferner gewisse oxydierende Fermente (indirekte: Oxydasen) treten.

Sie konnten Blut in einer 100000 fachen Verdünnung vermittelst der Benzidinprobe nachweisen. Hierza ist zu bemerken, dass sie defibriniertes Kaninchenblut als eine $0,001^{\%} \%$ ige Suspension in destilliertem Wasser benutzten.

Dabei führen sie noch zum Vergleich an, da ss die Teichmann'sche Probe in einer 20000 fachen Verdünung, cae G u a a kprobe nur in einer 5000 fachen Verdünnung positiv ausfälli. Die Grenze der Brauchbarkeit der spektroskopischen Methode geben sie nicht an, doch dürfte dieselbe in einer 2000 fachen Verdünnung ihre Grenze finden. Seit der Zeit, d. h. während einem Dezennium, ist die alkoholische Benzidinlősung zum qualitativen Nachweis von Oxydationsfermenten sowohl in Pflanzensäften wie in Milch, Blut usw. verwendet, sowie zum Blutnachweis in Harn, Fäces u. a. m. herangezogen worden. Nähere Literaturangaben hierüber finden sich in der Arbeit von Batelli und Ster $\mathbf{n}$ in den Ergebnissen der Physiologie ${ }^{\mathbf{1}}$ ).

Dagegen ist eine wässrige Benzidinlösung, soweit ich die Literatur überblicken kann, nur bei zwei Autoren bei den biologischen Oxydationsreaktionen zur Anwendung gekommen, uämlich bei Madelung ${ }^{2}$ ) und B egemann ${ }^{3}$ ). Beide Autoren strebten danach, eine Methode zur quantitativen Bestimmung der Oxydationsfermente vermittelst Benzidin als Reagens ausfindig zu machen.

Madelung stellte sich eine Benzidinlösung aus der Base in der Weise her, dass er eine heissgesättigte Benzidinlösung nach dem Erkalten filtrierte und das Filtrat, das, wie Madelung angibt, etwa $0,04 \%$ Benzidin enthielt, als Chromogen verwendete.

Begemann dagegen benutzte eine bei $45^{\circ} \mathrm{C}$. gesättigte Benzidinchlorhydratlösung.

Jene Benzidinlösungen sind am Ende dieser Arbeit in bezug auf ihre Farbenaktivität zum Vergleich mit meiner Benzidinlösung heran-

1) Batelli und Stern, Die Oxydationsfermente. Ergebn. d. Physiol. Bd. 12 S. 96. 1912.

2) Madelung, Über die Beziehungen der Hämoglobinderivate und P'eroxydasen zu anorganischen Katalysatoren. Zeitschr. f. physiol: Chemie Bd. 71 S. 204.1911.

3) Begemann, Beiträge zur Kenntnis pflanzlicher Oxydationsfermente. Inaug.-Diss. Bern 1915. 
gezogen worden. I nannten Autoren hier nicht weiter ein, weil sie die in dieser Arbeit auftauchenden Fragen nicht berühren, sondern verweise auf die Originalarbeiten.

An die Erfahrutigen Begeman p's bei seinem „Reagenzglasverfahren" mit Benziọin zur quantitativen Bestimmung pflanzlicher Peroxydasen anknüpfe nd, benutzte 'sh ich bei den biologischen Oxydationsreaktionen tes Blutes im $x$ stem Blut-Benzidin $-\mathrm{H}_{2} \mathrm{O}_{2}$ und bei einigen Metallsalzversuchen dos Benzidinchlorhydrat in bei $45^{\circ} \mathrm{C}$. gesättigter ,Lösung.

$\mathrm{Zu}$ meiner Verfugung stand ein Präparat Benzidinum hydrochlor. purum (Merek), das leur bei d₹̣n Vorversuchen henutzt wurde.

Später habe ich alls meine Untersuchungen mit einem gleichbenannten Benzidin von Gribler in Leipzig ausgeführt.

Die ersten Versuche mit der bei $45^{\circ}$ C. gesättigten Benzidinchlorhydratlösung fielen scheinbar gesetzmässig und konstant, auch betreffs der Farbenintensität, aus.

Doch eines Tages fiel die Farbenreaktion eines Eisenoxydpräparates in einer enormen Verdünnung (1:30000000 auf Eisenoxyd bezogen) positiv aus. Gleichzeitig hatte ich bemerkt, dass die bei $45^{\circ} \mathrm{C}$. gesättigte Benzidinlösung beim Erkalten über Nacht in langen dicken Nadeln, die an der Wand des Reagenzglases mit der Basis aufsitzend, in die Flüssigkeit hineinragten, auskristallisiert war.

Diese Kristalle, bei $45^{\circ} \mathrm{C}$. wieder gelöst, gaben diese mit Eisenoxyd $+\mathrm{H}_{2} \mathrm{O}_{2}$ unerwartet intensive Farbenreaktion.

Die "Sprünge" in der Farbenreaktion sind auch andern Autoren aufgefallen, doch haben sie diese Erscheinung entweder einer der andern Komponenten des Systems zugeschrieben oder nicht weiterverfolgt. Auch bei den andern Chromogenen, die bei den biologischen Oxydationsreaktionen in noch grösserem Umfang als das Benzidin Anwendung gefunden haben, sind von nambaften Forschern auf diesem Gebiete ähnliche Beobachtungen verzeichnet worden. Hier war es aber auch in der Regel nur das Ferment, das für die Stärke der Farbenreaktion verantwortlich gemacht wurde. Eine Ausnahme unter diesen Autoren ist $\mathrm{Bach}$.

Schon im Jahre 1912 publizierte Bach zusammen mit Maryanowitsch ${ }^{1}$ ) eine Arbeit „Zur Kenntnis der Spezifitäts-

1) Bach und Maryanowitsch, Zur Kenntnis der Spezifitätserscheinungen bei der Phenolasewirkung. Biochem. Zeitschr. Bd. 42 S. 417. 1912. 
erscheinungen bei der Phenolasewirkung". Leider wurde ich erst nach Fertigstellung meiner Arbeit auf diesen Aufsatz aufmerksam gemacht. Dass die Arbeit Bach's und Maryanowitsch's mir entgangen ist, ist damit zu erklären, dass sie nichts über das Benzidin als Chromogen enthält, und dass ich mich anderseits nur mit dem Benzidin als Chromogen bei den biologischen Oxydationsreaktionen befasst und dementsprechend die Literatur durchgesehen habe.

Bach und Maryanowitsch benutzten Guajakol, Hydrochinon; Orcin, Pyrogallol, $\alpha$-Naphtol + Paraphenylendiamin als Chromogene und studierten die Phenolasewirkung, d. b. die Oxydation dieser Substrate durch die Phenolase des Pilzes Lactarius vellereus in Gegenwart von Calciumchlorid, Calciumacetat, Zinksulfat, Zinkacetat, Magansulfat, Maganacetat und Aluminiumsulfat.

Indem sie bei dem gleichen Substrat die zugesetzten Salze änderten oder bei dem gleichen Salz verschiedene Substrate prüften, fanden sie, "dass für die Beeinflussung der Phenolasewirkung durch Metallsalze die Natur der zu oxydierenden Substrate maassgebend ist". Sie sprechen weiter den untersuchten Substraten bzw. Chromogenen Spezifitäten zu, eine Ansicht, die sie durch das Nichtauffinden spezifisch wirkender Phenolasen bestätigt glauben.

Sie präzisieren damit den Angriffspunkt der Salzeinwirkung bei den biologischen Oxydationsreaktionen. Aber sie führen gleichzeitig einen neuen, unbekannten Faktor in das Oxydationssystem ein, ohne den der Aufklärung harrenden Kernpunkt bei den biologichen Oxydationsreaktionen, die chemische Konstitution des Substrates und seines Oxydationsproduktes, zu treffen oder ihm näher zu kommen.

Den ersten Versuch, sowobl der Farbenreaktion bei den biologischen Oxydationsreaktionen wie den bei diesen beobachteten übrigen Erscheinungen und Widersprüchen eine chemisch definierbare Basis zu geben, hat unzweifelhaft Fräulein Dr. Woker ${ }^{1}$ ) in ibrer "Theorie der Benzidinoxydation" unternommen.

Zwei weitere Arbeiten von $\mathrm{Bach}$, die nach Abschluss meiner Arbeit erschienen sind, will ich an dieser Stelle noch nachträglich erwähnen, weil sie den Einfluss der chemischen Konstitution des Substrates auf die Farbenintensität bei den biologischen Oxydationsreaktionen beleuchten.

1) Gertrud Woker, Die Theorie der Benzidinoxydation. Ber. d. deutsch. chem. Gesellsch. Bd. 49 S. 2319-2337, 1916, Bd. 50 S. 672-677. 1917. 
In der ersten Arbeit ${ }^{1}$ ) mit der Frage "Kommt Peroxydase in Hefen vor?" stellte Bach fest, dass die von Harden und Silva beobachtete Peroxydasewirkung der Hefe bei Anwendung des p-Phenylendiamins in Gegenwart von $\mathrm{H}_{2} \mathrm{O}_{2}$ auf den Säuregehalt der Hefe zurückzuführen sei. $\mathrm{Bach}$ konnte nachweisen, dass eine Violettfärbung im System $\mathrm{HCl}-\mathrm{p}$-Phenylendiamin $-\mathrm{H}_{2} \mathrm{O}_{2}$ ohne Hefe auftrat, und dass das Maximum der Violettfärbung erreicht wurde, wenn das Verhältnis von $\mathrm{p}$-Phenylendiamin $\mathrm{zu} \mathrm{HCl}$ sich wie $1: 0,5$ verhielt.

In der zweiten Arbeit $\mathrm{Bach}$ 's ${ }^{2}$, die die Reaktion einer durch Ultrafiltration gereinigten Peroxydase behandelt, findet sich eine interessante Beobachtung, die sich wieder auf das Substrat bezieht. $\mathrm{Bach}$ fand, dass das 0-Kresol und seine Derivate, Saligenin, Salicylaldehyd und Salicylsäure, sich gegenüber Peroxydase $\mathrm{H}_{2} \mathrm{O}_{2}$ verschieden verbielten. So wurden 0 -Kresol und Saligenin bei derselben Farbenreaktion von Peroxydase- $\mathrm{H}_{2} \mathrm{O}_{2}$ glatt oxydiert, dagegen der Salicylaldehyd erst, nachdem das System alkaliseh gemacht worden war, und die Salicylsäure wurde überhaupt nicht wahrnehmbar angegriffen, weder in saurem noch in neutralem oder alkalischem Milieu.

Äbnlich verhielten sich das $\mathrm{p}$-Kresol und seine Derivate. Nitrogruppen sowohl in Ortho- wie in Parastellung in das Phenol eingeführt, verhinderten jede Oxydation durch das System Peroxydase- $\mathrm{H}_{2} \mathrm{O}_{2}$. Da nun für meine Untersuchungen eine Benzidinlösung von konstanten Eigenschaften unumgänglich war und nach meiner Ansicht eine weitere erfolgreiche Bearbeitung der Oxydationsfermente von dem Besitz eines einfachen und konstanten Chromogens abbängig ist, wurde zur Herstellung eines solchen geschritten.

Ich griff zuerst zum Mikroskop und konnte feststellen, dass das käufliche Benzidinchlorhydrat aus einem Gemenge heterogener Kristalle, und zwar von Blättchen und Säulen oder Stäbchen in wechselnder Grösse und ungleichmässiger Ausbildung, bestand. Die bei $45^{\circ}$ gesättigte Lösung dieses Benzidins war farbenaktiv, d. b. das Benzidinchlorhydrat liess sich leicht durch Oxydation in Benzidinblau überführen. Das Benzidinblau bestand aus blaugefärbten bieg-

1) Bach, Kommt Peroxydase in Hefen vor? Fermentforschung Bd. 1 S. 197.1915.

2) Bach, Sur les réactions de la peroxydase purifiée par nltrafiltration. Arch. des sciences phys. et nat. t. 42 p. כ̌6. 1916. 
samen Nadeln, die, wenn ausserdem Blättchen anwesend waren, im mikroskopischen Bilde aus und längs der Längsseite eines Blättchens emporzuwachsen schienen.

Weiter konnte ich beobachten, dass der Bodenkörper der gesättigten Lösung (im Reagenzglase oder im Kolben) in bezug auf das Verhältnis zwischen den verschiedenen Kristallformen sich veränderte. Nach mehrmaligem Erwärmen und Erkalten der gesättigten Lösung trat die "Stäbchen"form der Kristalle zurück, und die Blättchen nahmen im mikroskopischen Bilde zu. Aber, und das ist das Interessanteste, wurde gleichzeitig zu der gesättigten Lösung inkl. Bodenkörper beim Erwärmen Wasser zugefügt, so trat beim Erkalten auch die „Stäbchen "form zurück. Dafür erschien eine neue Kristallform, die Nadeln, neben den vorhin erwähnten beiden ursprünglichen Krisfallformen, eine Umwandlung, die auch makroskopisch an dem Kristallgemenge in der Flüssigkeit deutlich zu sehen war (beim Zurücktreten der Stäbchen und Auftreten der Nadeln). In den beiden Fällen nahm die Farbenaktivität zu, im zweiten am stärksten. Die Nadeln waren, wie die Stäbchen, leicht löslich.

Da ich eine Aufklärung über die konstitutive Eigenart des Benzidins noch nicht besass, ging ich zunächst empirisch vor, um die Ursache der wechselnden Eigenschaften desselben, deren Kenntnis für die biologischen Oxydationsreaktionen als eine conditio sine qua non angesehen werden muss, näher kennenzulernen.

Danach zog ich ein paar Lehrbücher der Chemie zu Rate.

$\mathrm{K}_{\mathrm{rafft}^{1}}{ }^{1}$ ) sagt in seinem Lehrbuche vom Benzidin bei Besprechung der Herstellung desselben: "Man stellt es in grösseren Mengen dar, indem man Azobenzol $\mathrm{C}_{6} \mathrm{H}_{5}-\mathrm{N}=\mathrm{N}_{-} \mathrm{C}_{6} \mathrm{H}_{5}$ in stark saurer Lösung reduziert, wobei das zunächst entstandene, nicht salzbildende Hydrazobenzol $\mathrm{C}_{6} \mathrm{H}_{5-} \mathrm{NH}_{-} \mathrm{NH}_{-} \mathrm{C}_{6} \mathrm{H}_{5}$ durch die benutzte starke Säure eine eigentümliche Unwandlung in das basisehe Benzidin $\mathrm{NH}_{2}-\mathrm{C}_{6} \mathrm{H}_{4-}$ $\mathrm{C}_{6} \mathrm{H}_{4-} \mathrm{NH}_{2}$ (neben Diphenylin) erleidet! Benzidin kristallisiert aus heissem Wasser in grossen glänzenden Blättchen, Schmelzpunkt $122^{\circ}$. C. zweisäurige Base. Chlorhydrat $\mathrm{C}_{12} \mathrm{H}_{12} \mathrm{~N}_{2}-2 \mathrm{HCl}$, in -Wasser leicht lösliche rhombische Tafeln."

Im Lehrbuch der Mikrochemie von Professor Emich ${ }^{2}$ ) finde ich folgende Angabe über das Benzidin. Emich schreibt: „Benzidin

1) Krafft, Lehrbuch der organischen Chemie, 4. Aufl, S. 585.

2) Emich, Lehrbuch der Mikrochemie S. 178. 1911. 
a) Farblose Blättchen, in Wasser sehr schwer löslich, löslich in Alkohol und Äther. Salzsäure bildet zwei Chlorhydrate: das eine (mit $2 \mathrm{HCl}$ ) entsteht aus der Base und wenig konzentrierter Salzsäure, das andere scheidet sich eventuell bei Zusatz von Wasser (in Nadeln) ab."

Diese beiden Angaben und meine oben angegebenen Beobachtungen über die allmähliche Umwandlung des Bodenkörpers der gesättigten wässrigen Benzidinlösung, sowohl nach der Form wie nach den Eigenschaften, wurden für mich der Ausgangspunkt folgender Überlegung.

Die Blättchen sind die Kristallform der Benzidinbase, die in Wasser fast unlöslich ist, die Stäbchen wahrscheinlich das Dichlorhydrat und die Nadeln das Monochlorhydrat. Sie sind leicht löslich in Wasser. Nach allem war die letzte Kristallform die günstigste für meinen Zweck.

Ich unternahm deshalb den Versuch, aus Benzidinbase ein Monound Dichlorid durch Znfügung einer berechneten Menge $\mathrm{HCl}$ herzustellen, um dann mit den beiden Benzidinchlorhydraten Vergleiche in bezug auf die Farbenreaktion mit ein und demselben Oxydationsmittel, in diesem Falle Blut $+\mathrm{H}_{2} \mathrm{O}_{2}$, anstellen zu können.

Aber es gelang mir weder das Mono- noch das Dichlorid auf diesem Wege willkürlich herzustellen. Die Produkte, die ich aus der Benzidinbase erbielt, und ihre Eigenschaften sind in der auf S. 325 abgedruckten Tabelle zusammengestellt.

In der Absicht, ein Benzidindichlorhydrat herzustellen, wurden im ersten Versuch I 2 g Benzidinbase mit 4 cem einer $25 \%$ igen HCl-Lösung unter Wasserzusatz gekocht, ohne dass eine vollständige Lösung gelang, und filtriert. Der auf dem Filter bleibende Rückstand ist mit Ia, der aus dem Filtrat beim Erkalten auskristallisierte Teil mit I $b$ bezeichnet.

Die physikaliseh-chemischen Eigenschaften des Rückstandes Ia sind :

Kristallform: rhombische Blättchen, Schmelzpunkt $341^{\circ}$ C. nach vorangegangener schwärzlicher Zersetzung von $160^{\circ} \mathrm{C}$. an, Wasserlöslichkeit in 100 facher Verdünnung bei $20^{\circ} \mathrm{C}$. fast Null. Azidität der Lösung schwach. (1 cem $\left.=0,9 \mathrm{cem} \frac{\mathrm{n}}{100}-\mathrm{NaOH}\right)$. Aktivität $=$ Benzidinblaureaktion) relativ am stärksten. 


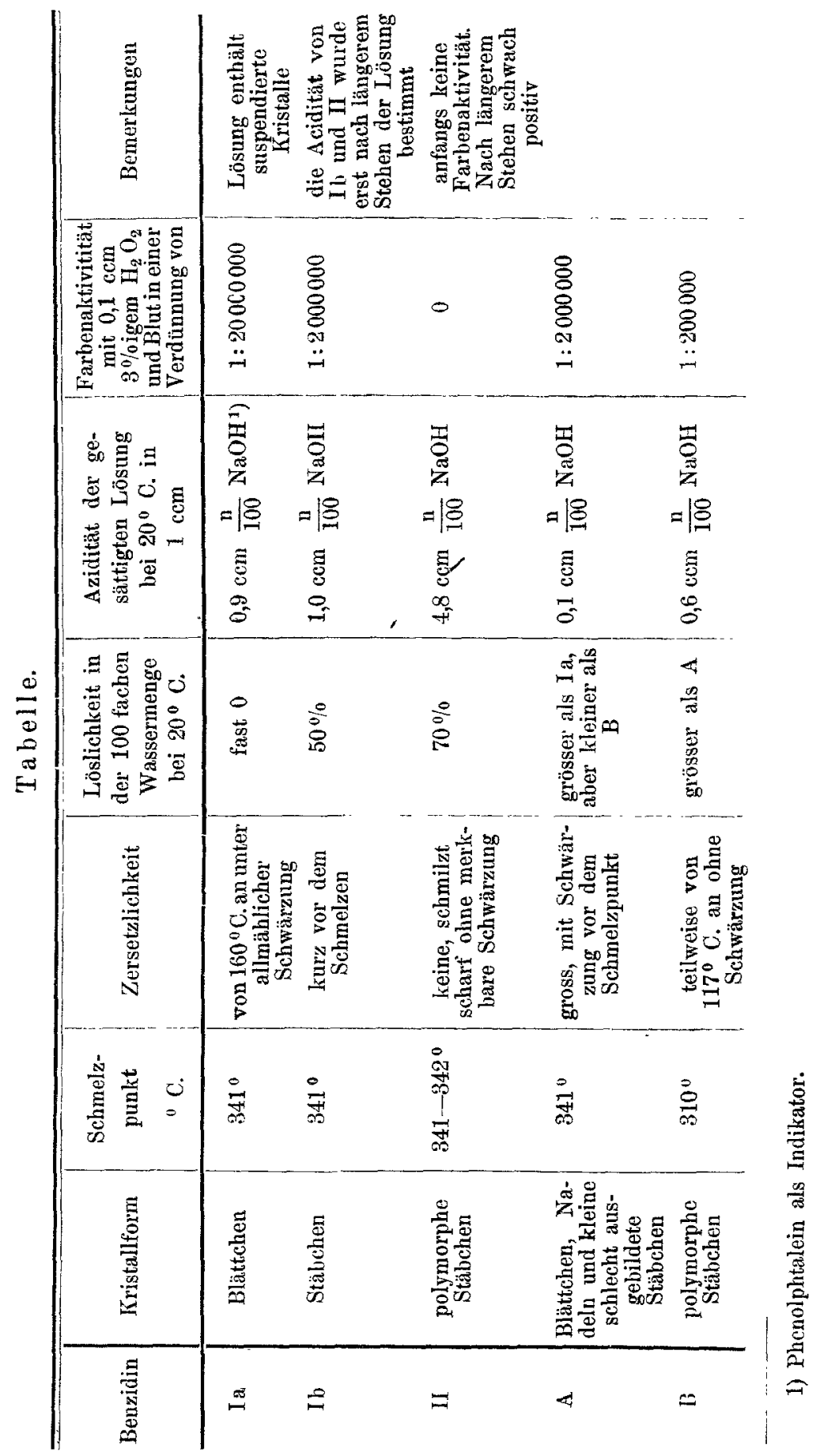


Die Eigenschaften der Kristalle des Filtrats Ib sind :

Kristallform: Stäbchen, Schmelzpunkt $341^{\circ} \mathrm{C}$., Zersetzung erst kurz vor dem Schmelzen, Wasserlöslichkeit bei $20^{\circ} \mathrm{C}$. in der 100 fachen Wassermenge $50 \%$. Azidität der Lösung etwas stärker als im vorigen Fall $\left(1 \mathrm{ccm}=1,0 \mathrm{ccm} \frac{\mathbf{n}}{100}-\mathrm{NaOH}\right)$. Alktivität schwächer wie die des Rückstandes.

Um nun anderseits zu einem Benzidinmonochlorbydrat zu gelangen, wurde ähnlich verfahren, nur mit dem Unterschied, dass zu $2 \mathrm{~g}$ Benzidinbase $2 \mathrm{ccm} 25 \%$ ige HCl-Lösung anstatt $4 \mathrm{ccm}$ gesetzt wurden. Die Misehung mit Wasser, bis zu vollständiger Lösung versetzt, wurde hierauf filtriert. Es blieb kein Rückstand auf dem Filter zurück.

II ist das beim Erkalten aus dem Filtrat auskristallisierte Benzidin. Seine Kristalle zeigten gegenüber I $b$ folgende Eigenschaften:

Kristallform: polymorphe Stäbchen, Schmelzpunkt $341-342^{\circ} \mathrm{C}$. ohne vorherige Zersetzung, Wasserlöslichkeit in 100 facher Verdünnung bei $20^{\circ} \mathrm{C} .70 \%$. Azidität sebr stark $\left(1 \mathrm{ccm}=4,8 \mathrm{ccm} \frac{\mathrm{n}}{100}-\mathrm{NaOH}\right)$. Aktivität Null im Anfang, nach längerem Stehen schwach positiv. Sämtliche drei Produkte inkl. der Mutterlauge enthielten Chlor (Beil stein'sche Probe).

Es wurde noch ein Versuch, aber mit normaler Salzsäure angestellt um das Monochlorhydrat aus der Benzidinbase zu gewinnen. $1 \mathrm{~g}$ Benzidinbase wurde in $11,85 \mathrm{~g} \mathrm{n}$. $\mathrm{HCl}$ eingetragen. $10,85 \mathrm{~g} \mathrm{HCl}$ ist die für ein Dichlorhydrat berechnete nötige Menge Salzsăure. Hierzu kam noch $1 \mathrm{~g} \mathrm{n}$. $\mathrm{HCl}$ als Überschuss. Die Misebung wurde gekocht und dann bis zur vollständigen Lösung Wasser hinzugefügt.

Nun wurde, um ein Monochlorhydrat zu erhalten, der berechnete Überschuss plus ein $\mathrm{HCl}$ mit $6,4 \mathrm{cem}$ n. $\mathrm{NaOH}$ neutralisiert und kochendheiss filtriert.

A bezeichnet den Filterrückstand, B die Kristalle aus dem erhaltenen Filtrat. Rückstand, Filtrat und Mutterlauge zeigten mit der Beilst e in'schen Probe einen Chlorgehalt, jedoch der Rüekstand den geringsten.

Die physikaliseh-chemischen Merkmale der Kristalle dieses Rückstandes und Filtrates waren:

Für den Rückstand A :

Kristallform: polymorphe Blättchen, Nadeln und kleine, schlecht 
ausgebildete Stäbchen, Schmelzpunkt $341^{\circ} \mathrm{C}$. mit starker vorausgehender schwärzlicher Zersetzung Wasserlöslichkeit grösser als I a, aber kleiner als B. Azidität sehr sehwach $\left(1 \mathrm{cen}=0,1 \mathrm{cem} \frac{\mathrm{n}}{100}-\mathrm{NaOH}\right)$. Farbenaktivität gross.

Für die Kristalle aus dem Filtrat:

Kristallform: polymorphe Stäbchen und kleine Blättchen, Schmelzpunkt $310^{\circ} \mathrm{C}$. mit teilweiser Zersetzung von $117^{\circ} \mathrm{C}$. an ohne Schwärzung. Wasserlöslichkeit grösser als A (Rückstand). Azidität mittelstark $\left(1 \mathrm{cem}=0,6 \mathrm{ccm} \frac{\mathrm{n}}{100}-\mathrm{NaOH}\right)$. Farbenaktivität mässig.

Ich lasse noch fünf Serien Hämoperoxydaseversuche in Gegenwart von $\mathrm{H}_{2} \mathrm{O}_{2}$ mit den oben beschriebenen fünf Benzidinchlorhydraten als Chromogene folgen. Diese Versuche zeigen mit Deutlichkeit, wie wichtig die Kenntnis des Chromogens für Schlüsse über die Peroxydasewirkung ist.

Für jede der fünf Serien, aus je fünf Reagenzgläsern bestehend, kam eins der fünf Benzidinchlorhydrate zur Anwendung. Die Reagenzgläser jeder Serie wurden mit den gleichen fallenden Mengen Menschenblut in $1 \mathrm{ccm}$ Aqua dest. beschickt, jedem Glase $1 \mathrm{ccm}$ bei $45^{\circ} \mathrm{C}$. gesättigter Benzidinlösung der entsprechenden Serie zugefügt und die Farbenintensität in Gegenwart von $0,1 \mathrm{ccm} 3 \%$ igem $\mathrm{H}_{2} \mathrm{O}_{2}$ nach 1 Minute notiert.

Die Farbenreaktionen boten folgendes Bild:

\begin{tabular}{l|c|c|c|c|c}
\hline 0,1 ccm $3 \%$ iges $\mathrm{H}_{2} \mathrm{O}_{2}+$ & 0,0001 & 0,00001 & 0,000001 & 0,0000001 & 0,00000001 \\
Blut in $1 \mathrm{ccm} \mathrm{Aq.}$ & & & & & \\
\hline Mit $1 \mathrm{ccm}$ Benzidin I a : & +++ & ++ & $+(+)$ & + & 0 \\
Mit $1 \mathrm{ccm}$ Benzidin Ib : & $+(+)$ & + & 0 & 0 & 0 \\
Mit $1 \mathrm{ccm}$ Benzidin II : & 0 & 0 & 0 & 0 & 0 \\
Mit $1 \mathrm{ccm}$ Benzidin A : & ++++ & $+(+)$ & + & 0 & 0 \\
Mit $1 \mathrm{ccm}$ Benzidin B : & ++++ & + & 0 & 0 & 0
\end{tabular}

Bemerkung: 1. In der ersten Serie waren suspendierte Benzidinkristalle, die sich tiefblau färbten und den Farbstoff an sich rissen. Die Blaufärbung war beständig ( 8 Stunden beobachtet). Diese fünf Serien zeigen ohne weiteres die einschneidende Bedeutung der Azidität und lassen den Einfluss des Herstellungsverfahrens des Benzidinchlorhydrats auf die Farbenreaktion erkennen.

Weiter deuten diese Versuche darauf hin, dass die qualitative Steigerung der Farbenreaktion durch die Anwesenheit von Blättchen in schwach saurem Medium möglich ist, aber dass ein wasserlösliches, 
konstantes Benzidin von hoher Farhenaktivität bei einem Benzidinmonochlorhydrat zu finden ist, resp. dort, wo sich das Benzidin zum $\mathrm{HCl}$ verhält wie $1: 1$.

Die Verdünnungsgrenze, auf eine Totalflüssigkeitsmenge von 2 ecm bezogen, war im ersten Falle (mit Benzidin Ia) 1:2000000 und im zweiten (mit Beuzidin A) 1:2000000.

Indem ich mir die Erkenntnis zwischen dem Zusammenhang von Azidität und Wasserlöslichkeit einerseits und von Farbenaktivität und Schwerlöslichkeit anderseits zunutze machte, ging ich jetzt daran, mir aus einem käuflichen Benzidinchlorhydrat die erwünschte Benzidinmonochlorhydratlösung empirisch herzustellen. Ich besass ein Benzidinum bydrocbloricum purum von Grübler in Leipzig. Von diesem stellte ich eine bei $20^{\circ} \mathrm{C}$. gesättigte Lösung her und bestimmte die Azidität dieser Lösung. $10 \mathrm{ccm}$ derselben wurden von $23 \mathrm{ccm}$ $-\frac{\mathrm{n}}{100}-\mathrm{NaOH}$ mit Phenolphtalein als Indikator neutralisiert. Hierbei fiel die Base aus.

Um nun die Azidität der ursprünglichen Benzidinlösung für die optimale Farbenintensität zu ermitteln, ging ich folgendermaassen weiter vor.

Fünf Kolben, mit 1, 2, 3, 4, 5 bezeichnet, wurden mit je $10 \mathrm{ccm}$ bei $20^{\circ} \mathrm{C}$. gesättigter Benzidinlösung beschickt und zu diesen steigende Mengen $\frac{\mathbf{n}}{100}-\mathrm{NaOH}$ zugefügt, und zwar zum ersten Kolben $0 \mathrm{ccm}$, zum zweiten 5 usw. 10, 15 und $20 \mathrm{ccm}$.

In fünf Reagenzgläsern, ebenfalls mit $1,2,3,4,5$ bezeichnet, befanden sich je $0,0001 \mathrm{ccm}$ Blut in $1 \mathrm{ccm}$ Aqua dest.

Diesen Gläsern wurde $1 \mathrm{ccm}$ Lösung von den gleich bezeichneten Kolben zugegeben und hierauf $0,1 \mathrm{ccm} 3 \%$ iges $\mathrm{H}_{2} \mathrm{O}_{2}$ zugesetzt.

Die Farbenintensität war nach 3 Minuten folgende:

Glas $\begin{array}{ccccc}1 & 2 & 3 & 4 & 5 \\ +(+) & ++ & ++++ & ++ & +\end{array}$

Dieses Ergebnis zeigte mir, dass ich im Glase 3, dort, wo ich die Azidität meiner Benzidinlösung um fast die Hälfte heruntergedrückt hatte, die stärkste Farbenreaktion erreichte.

Damit war die Einstellung auf $1 \mathrm{HCl}$ im dritten Kolben noch nicht genau, und da mir für die Wegneutralisation des zweiten $\mathrm{HCl}$ eine genaue $\frac{n}{100}-\mathrm{NaOH} \cdot$ Lösung zur Verfügung stand, wollte ich mir vor der 
Haud eine $\frac{\mathbf{n}}{100}$-Benzidinchlorhydratlösung herstellen und in dieser dann exakt das eine $\mathrm{HCl}$ wegneutralisieren, um später, wenn möglich, zu einer höheren Konzentration überzugehen.

Das Molekulargewicht des Benzidinchlorhydrats ist nach der Formel $\mathrm{C}_{12} \mathrm{H}_{12} \mathrm{~N}_{2} \cdot 2 \mathrm{HCl}$ gleich 284 . Um $100 \mathrm{cem}$ einer $\frac{\mathfrak{n}}{100}$-Lösung. zu erhalten, löste ich deshalb $0,284 \mathrm{~g}$ Benzidinchlorhydrat (Grübler) in $100 \mathrm{cem}$ destilliertem Wasser auf. Die Auflösung des Benzidins in Wasser wird durch Erwärmen bescbleunigt, ohne dass dasselbe in dieser Konzentration beim Erkalten wieder ausfällt. $10 \mathrm{cem}$ dieser Lösung hatten eine Azirlität von $20 \mathrm{ccm} \frac{\mathrm{n}}{100}-\mathrm{NaOH}-\mathrm{Lösung}$. Also ist meine vorhin erwähnte bei $20^{\circ} \mathrm{C}$. gesättigte Benzidinchlorhydratlösung nicht viel konzentrierter gewesen, ein Umstand, der zeigte, dass eine wasserlösliche, konstante Benzidinchlorhydratlösung für meinen Zweck kaum konzentrierter hergestellt werden konnte. Diese Annahme ist auch durch spätere Versuche bestätigt worden.

In $100 \mathrm{ccm} \frac{\mathrm{n}}{100}$-Benzidinchlorhydratlösung wurde nun das eine $\mathrm{HCl}$ mit 100 cem $\frac{\mathbf{n}}{100}-\mathrm{NaOH}$-Lösung wegneutralisiert, ohne dass Benzidinkristalle ausfielen. Bei über diese Grenze fortgesetzter Neutralisation trat dagegen ein Auskristallisieren des Benzidins auf. Mit der $\frac{\mathrm{n}}{100}$-NaOH-Lösung hatte ich allerdings auch die Konzentration meiner Benzidinchlorhydratiösung auf $\frac{\mathrm{n}}{200}$ herabgesetzt. $10 \mathrm{ccm}$ dieser Benzidinlösung wurden von $5 \mathrm{ccm}$ einer $\frac{\mathrm{n}}{100} \mathrm{NaOH-Lösung} \mathrm{neutra-}$ lisiert. Das war nicht zu vermeiden, denn eine Reihe von Versuchen, die ich hier nicht wegen ihrer negativen Ergebnisse niederschreibe, aber die da hinausgingen, das eine $\mathrm{HCl}$ ohne eine gleichzeitige Herabsetzung der Konzentration der. Benzidinlösung zu entfernen, schlugen fehl.

Bevor ich noch auf die Empfindlichkeit und auf die Haltbarkeit des Benzidins im allgemeinen und meiner $\frac{\mathfrak{n}}{200}$-Benzidinmonochlorhydratlösung eingehe, möchte ich noch einen Vergleich der Farbenaktivität dieser Lösung mit wasserlöslichen Benzidinlösungen anderer 
Autoren anstellen. Ich meine die in der Einleitung erwähnten von Madelung und Begemann.

Die Anwendbarkeit der bei $45^{\circ} \mathrm{C}$. gesättigten Benzidinchlorbydratlösung Begemann's geht aus dem vorigen deutlich hervor, war sie doch der Ausgangspunkt meiner Peroxydaseversuche, und ihre Inkoustanz bei biologischen Oxydationsreaktionen gab eben den Anlass zu den hier niedergelegten Untersuchungen.

Die Farbenaktivität jener Benzidinlösung kann sowohl diejenige meiner Benzidinlösung übertreffen wie auch anderseits hinter dieser zurückbleiben, je nach dem Herstellungsverfahren des Benzidinchlorhydrats, wie meine Versuche deutlich zeigen.

Die Benzidinlösung: Madelung's habe ich mit der meinigen verglichen und konnte für jene eine doppelt so grosse Farbenaktivität, bezogen auf die Verdünnungsgrenze derselben Blutlösung, feststellen. Allerdings trat die Farbenreaktion erst nach tropfenweisem, vorsichtigem Zusatz von $\mathrm{HCl}$ oder $3 \%$ iger Essigsäure auf. Einen Einfluss einer $0,9 \%$ igen $\mathrm{NaCl}$-Lösung auf die Farbenintensität an der Verdünnungsgrenze der Blutlösung konnte ich nicht, wie Madelung. konstatieren. Diese Befunde stehen vollkommen in Einklang mit dem von mir'festgestellten Zusammenhang zwischen Farbenaktivität und Schwerlöslichkeit, nur ist hier die Farbenintensität von den Mengen Säure, die zugesetzt werden, abhängig, und das farbenaktive Benzidinsalz ist im System gar nicht vorgebildet vorhanden.

$\mathrm{Ob}$ die Bildung dieses Salzes in Gegenwart von Peroxydase- $\mathrm{H}_{2} \mathrm{O}_{2}$ unbeeinflusst von diesen vor sich geht, ist zweifelhaft. Trotz ihrer Farbenaktivität kann die Benzidinlösung Madelung's wie diejenige Begemann's meiner Ansicht nach bei den biologischen Oxydationsreaktionen für quantitative $Z$ weeke nicht als geeignetes Reagens angesehen werden.

Das Alter hat, wie schon im Anfang dieser Arbeit erwähnt wurde, auf die wässrige Benzidinchlorhydratlösung einen merkbaren Einfluss. Da die Faŕbenaktivität am grössten ist, wenn im Benzidinmolekül nur ein $\mathrm{HCl}$ vorhanden ist, so dürfte diese Erscheinung von einem Salzsäureverlust herrühren, wodurch das Benzidinsalz von grösserer Farbenaktivitit entsteht.

Dass höhere Temperaturen diese "Reifung" der Lösung begünstigen, braucht keine weitere Erklärung.

Mit dem Altern der Lösung trat auch eine sichtbare Veränderung auf. Die Lösung wurde schwärzlich, und es setzten sich grau-rötliche, 
fadenförmige Flocken auf den Boden des Kolbens oder Reagenzglases, doch blieb die Farbenaktivität meiner $\frac{\mathrm{n}}{200}$-Benzidinchlorhydratlösung wochenlang unverändert.

Ich habe auch Farbenreaktionsversuche mit dem System BlutBenzidin- $\mathrm{H}_{2} \mathrm{O}_{2}$ bei variierenden Temperaturen, und zwar bei $20^{\circ}, 40^{\circ}$, $60^{\circ}$ und $80^{\circ} \mathrm{C}$, angestellt. Das Optimum der Farbenreaktion war bei $40^{\circ} \mathrm{C}$. Die höheren Temperaturen setzten die Farbenintensität (blau), die niederen die Reaktionsgeschwindigkeit herab. Doch ist anzunehmen, dass hier die Hämoperoxydase von den höheren Temperaturen geschwächt worden ist. Die Reaktionsgeschwindigkeit bei der böchsten Temperatur war enorm gesteigert und kaum messbar. Das Benzidinblau durehlief alle Oxydationsstufen von blau, rot oder gelb bis schwarz in wenigen Sekunden.

Die Farbenaktivität der Benzidinlösung wird von Säuren und Alkalien. schon in Spuren merkbar beeinflusst. Diese Erscheinung bedarf einer ausführlichen Besprechung, weil mir nach Erkenntnis nachfolgender Tatsacheu die Frage berechtigt erscheint, ob nicht die durch Säuren und Alkalien verursachten Hemmungen oder Aktivierungen der biologischen Oxydationsreaktionen, wobei immer das Ferment als Angriffspunkt bezeichnet worden ist, bei einem andern Faktor des Systems, dem Chromogen, gesucht werden sollten?

Dass der Einfluss von Säure oder Alkali im System Blut-Benzidin$\mathrm{H}_{2} \mathrm{O}_{2}$ bei der Oxydationsreaktion nur beim Chromogen and nicht bei der Peroxydase zum Ausdruck kommt, ist, nachdem die Eigenschaften der wässrigen Benzidinlösung aufgeklärt worden sind, leicht verständlich und auch deutlich nachzuweisen.

Der Einfluss der Säure oder des Alkali ist natürlich nicht immer derselbe, sondern je nach dem Aziditätsgrad der Lösung verschieden. So bewirkt die Säure eine Steigerung der Farbenaktivität einer zu schwach sauren oder theoretisch neutralen Lösung ebenso, wie das Alkali die Farbenaktivität einer stark sauren Lösung begünstigt. Für eine $\frac{\mathbf{n}}{200}$-Benzidinmonochlorhydratlösung gilt, dass sowohl Säure wie Alkali schon in Spuren ihre Farbenaktivität herabsetzen. Dabei ist die Einwirkung des Alkali grösser als die der Säure. So setzte ein Tropfen $\frac{\mathbf{n}}{20}-\mathrm{NaOH}-\mathrm{Lösung}$ die Verdünnungsgrenze des Blutes bei der Oxydationsreaktion im System Blut-Benzidin- $\mathrm{H}_{2} \mathrm{O}_{2}$ um die Hälfte 
herab, während ein Tropfen $\frac{\mathrm{n}}{10}-\mathrm{HCl}$ obne Einfluss auf die Farbenintensität war. Die Einwirkung solch kleiner Mengen Natronlauge hängt natürlich auch von den sonstigen Mengenverhältnissen ab. Denn in einer Totalflüssigkeitsmenge von $2 \mathrm{cem}$ war nur $1 \mathrm{cem} \frac{\mathrm{n}}{200}$-Benzidinmonochlorhydratlösung vorhanden.

Die schon in dieser Arbeit früher betonte Tatsache des Einflusses der Säure und des Alkali auf die Oxydationsreaktion stellte mich weiter vor die Frage, ob die Säure resp. das Alkali nur in der Realstionsphase eingreift, oder ob sie schon vor der Reaktion eine Veränderung des Chromogens hervorgerufen hatte. Schon zu Beginn dieser Arbeit hatte ich beobachtet, dass in einer Benzidinchlorbydratlösung das Benzidin nicht beliebig mit Natronlauge ausgefällt und wieder durch $\mathrm{HCl}$-Zusatz in Lösung gebracht werden konnte, obne dass die Lösung durch mehrmalige Wiederholung dieser Prozedur definitiv inaktiv wurde. Dieser Befund findet auch seine Bestätigung in dem wechselnden Resultate bei dem Versuch durch berechnete Mengen Benzidinbase und $\mathrm{HCl}$ ein konstantes Produkt, das eine bestimmte Quantität $\mathrm{HCl}$ (z. B. als Monochlorhydrat) enthalten sollte, zu erzielen. Dies geht aus der Tabelle und den an diese sich anschliessenden Erläuterungen deutlich hervor.

Auch der folgende Versuch bestätigt obigen Befund. Ich brachte zunächst $1 \mathrm{ccm}$ einer bei $20^{\circ} \mathrm{C}$. gesättigten Benzidinchlorhydratlösung A (siehe Tabelle), die noch eine deutliche Farbenreaktion mit $0,00001 \mathrm{ccm}$ Blut, in Gegenwart von $0,1 \mathrm{ccm} 3 \%$ igem $\mathrm{H}_{2} \mathrm{O}_{2} \mathrm{gab}$, in je drei Reagenzgläser. Zu Glas 1 setzte ich $2 \times 0,5 \mathrm{ccm}$ Aqua dest., zu Glas 2 erst $0,5 \mathrm{ccm} \frac{\mathrm{n}}{100} \cdot \mathrm{NaOH}$ und hierauf $0,5 \mathrm{ccm} \frac{\mathrm{n}}{100} \cdot \mathrm{HCl}$ und zum dritten Glase dieselben Mengen wie zu Glas 2, aber in umgekehrter Reihenfolge. Die $\mathrm{NaOH}-$ und HCl-Lösungen waren aufeinander genau eingestellt.

Hierauf beschickte ich sämtliche drei Gläser mit $0,00001 \mathrm{cem}$ Blut und $0,1 \mathrm{ccm} 3 \%$ igem $\mathrm{H}_{2} \mathrm{O}_{2}$ und wartete die Farbenreaktion ab. Nur im Glase 1, d. h. im Kontrollglase, trat eine deutliche Farbenreaktion mit der Farbenintensität + auf. Diese drei Befunde sind eindeutig und stützen einander und gestatten den Schluss, dass das Benzidinchlorbydrat in der Lösung schon vor der Farbenreaktion eine chemische Veränderung erlitten, derzufolge es seine Reaktionsfähigkeit eingebüsst batte. 
Auch Salzlösungen können die Farbenreaktion des Systems BlutBenzidin $-\mathrm{H}_{2} \mathrm{O}_{2}$ beeinflussen, wobei in der Mehrzahl der Fälle ebenfalls das Chromogen der Angriffspunkt für das Salz ist.

So wird die Farbenreaktion gestört oder verhindert von Salzlösungen, die die Herabsetzung der Löslichkeitsgrenze des Chromogens herbeiführen, indem sie das Substrat ausfällen, oder es wird die Farbenintensität wie bei den Schwermetallsalzen dadurch gesteigert, dass diese selbst im System peroxydaseähnliche Eigenschaften entwickeln.

Diese Eigenschaften der Salzlösungen, die noch durch die Reaktion der Lösung in der einen oder andern Richtung gesteigert werden können, treten natürlich bei einer Benzidinlösung, die nur in einer Konzentration $\frac{1}{200}$ normal in Lösung gehalten werden kann, besonders oft und deutlich zutage.

Auch wäre noch an dieser Stelle die Einwirkung einer Kochsalzlösung auf das Benzidinblau besonders zu erwähnen. Das Kochsalz "konserviert" das Benzidinblau, indem es seine weitere Oxydation hemmt, wie schon vor mir Madelung feststellen konnte. Ist die Kochsalzlösung von höherer Konzentration als die Benzidinlösung, so fällt das Benzidin aus, wodurch in den böheren Blutkonzentrationen eine grössere Farbenintensität für kurze Zeit, vor dem Zusammenballen der Kristalle, vorgetäuscht wird. Das Benzidinblau wird ausgesalzen.

Die Verdünnungsgrenze der Blutlösung im System wird durch das Kochsalz nicht beeinflusst.

Für die theoretisch-chemische Erklärung, soweit sie nicht bei den in dieser Arbeit niedergelegten Befunden erwähnt worden ist, verweise ich auf die Arbeit von Fräulein Dr. Woker ${ }^{1}$ ) über die Theorie der Benzidinoxydation in den Berichten der Deutschen chemischen Gesellschaft.

Als Nichtfachmann habe ich in dieser Arbeit von jeder chemischen Erklärung Abstand genommen. Diese Aufgabe, die gleichzeitig meine Arbeit ergänzt, ist von der Leiterin des Instituts für physikalischchemische Biologie in Bern, Fräulein Dr. Woker, in liebenswürdiger Weise übernommen worden, wofür ich bier meinen verbindlichsten Dank gleichzeitig mit dem für ihr reges Interesse an dieser Arbeit ausspreche.

1) Gertrud Woker, Die Theorie der Benzidinoxydation. Ber. d. deutsch. chem. Gesellsch. Bd. 49 S. 2919-2337. 1916, Bd. 50 S. 672-677. 1917. 
334 M. Kjöllerfeldt: Beitrag zur Kenntnis des Benzidins als Chromogen usw.

\section{Zusammenfassung.}

Es wurde gezeigt:

1. dass die wässrige Benzidinchlorhydratlösung je nach dem Herstellungsverfahren des Benzidinchlorhydrats, dem Alter und der Behandlung der Lösung bei den biologischen Oxydationsreaktionen an Farbenaktivität verschieden ist;

2. dass zwischen Azidität und Wasserlöslichkeit einerseits und Schwerlöslichkeit und Farbenaktivität anderseits ein Zusammenhang besteht, und zwar nimmt die Azidität mit steigender Wasserlöslichkeit zu. Im Zusammenhang damit geht einer vermehrten Schwerlöslichkeit erhöhte Farbenaktivität parallel. Die Azidität hängt von dem Gehalt an hydrolytisch abgespaltener Salzsäure ab.

Auf Grund dieser Befunde habe ich ein Verfahren zur Herstellung einer $\frac{\mathbf{n}}{200}$-Benzidinmonochlorhydratlösung aus dem käuflichen Benzidinum hydrochloricum purum ausgearbeitet. In dieser Lösung ist das Benzidin zum $\mathrm{HCl}$ im Verhältnis $1: 1$ vorhanden. Die Lösung besitzt folgende Eigenschaften:

1. sie besitzt die grösstmögliche Konzentration bei niedrigster Azidität;

2. sie ist chemisch exakt darstellbar, sowie von hoher Farbenaktivität;

3. das Benzidin der Lösung wird von Salzlösungen, die eine höhere Konzentration besitzen als die der Benzidinlösung, ausgefällt, wodurch die Farbenreaktion direkt gestört oder verhindert wird;

4. Säure und Alkali in Spuren setzen ihre Farbenaktivität herab, das Alkali stärker wie die Säure;

5. ihre Farbenaktivität bleibt in verschlossener Flasche wochenlang unverändert. 